\title{
OPEN Behavior of magnetoelectric hysteresis and role of rare earth ions in multiferroicity in double perovskite $\mathrm{Yb}_{2} \mathrm{CoMnO}_{6}$
}

Jong Hyuk Kim, Ki Won Jeong, Dong Gun Oh, Hyun Jun Shin, Jae Min Hong, Jin Seok Kim, Jae Young Moon, Nara Lee ${ }^{\varpi}$ \& Young Jai Choi ${ }^{\bowtie}$

Double-perovskite multiferroics have been investigated because alternating orders of magnetic ions act as distinct magnetic origins for ferroelectricity. $\ln \mathrm{Yb}_{2} \mathrm{CoMnO}_{6}$, the frustrated antiferromagnetic order emerging at $T_{\mathrm{N}}=52 \mathrm{~K}$ induces ferroelectric polarization perpendicular to the $c$ axis through cooperative $\mathrm{O}^{2-}$ shifts via the symmetric exchange striction. In our detailed measurements of the magnetoelectric properties of single-crystalline $\mathrm{Yb}_{2} \mathrm{CoMnO}_{6}$, we observe full ferromagnetic-like hysteresis loops that are strongly coupled to the dielectric constant and ferroelectric polarization at various temperatures below $T_{\mathrm{N}}$. Unlike $\mathrm{Lu}_{2} \mathrm{CoMnO}_{6}$ with non-magnetic $\mathrm{Lu}^{3+}$ ions, we suggest the emergence of additional ferroelectric polarization along the $c$ axis below the ordering temperature of magnetic $\mathrm{Yb}^{3+}$ ions, $T_{\mathrm{Yb}} \approx 20 \mathrm{~K}$, based on the spin structure established from recent neutron diffraction experiments. While the proposed description for additional ferroelectricity, ascribed to the symmetric exchange striction between $\mathrm{Yb}^{3+}$ and $\mathrm{Co}^{2+} / \mathrm{Mn}^{4+}$ magnetic moments, is clearly given, anomalies of dielectric constants along the $c$ axis are solely observed. Our interesting findings on magnetoelectric hysteresis and the possible development of additional ferroelectricity reveal notable characteristics of double perovskites and provide essential guidance for the further examination of magnetoelectric functional properties.

Condensed-matter systems with strongly coupled order parameters offer immense opportunities for a fundamental understanding of their governing interactions as well as for the utilization of new technologies. Interesting cross-coupling effects can be observed in magnetoelectric and multiferroic materials, whose interlinked electric and magnetic order parameters have inspired materials research to explore new multifunctional materials and investigate the mechanisms underlying magnetoelectiricity ${ }^{1-4}$. Research has been aimed at discovering ferroelectricity driven by a specific type of ordered magnetic state. Ferroelectric distortions have been ascribed to both symmetric and antisymmetric parts of the magnetic exchange striction ${ }^{5,6}$. Because of this origin, the substantial coupling between structural distortions and magnetic order often results in a large variation in the dielectric and ferroelectric properties through the application of magnetic field $s^{2-4,7}$. Although various magnetic materials have been known to be magnetoelectrics or multiferroics, exploring new materials with cross-couplings is still beneficial for improving the feasibility of multiple functionalities.

Double-perovskite compounds, in which two different transition-metal ions are alternately located in octahedral environments, have been widely investigated. In such materials, combinations of mixed-valence magnetic ions reveal the intricate magnetic interactions and ionic valence/antisite disorders, which enable various fascinating physical properties, such as metamagnetism ${ }^{8-10}$, exchange bias ${ }^{11-13}$, magnetocaloric effect ${ }^{14-17}$, and multiferroicity ${ }^{18-21}$. Recent studies have also focused on the potential use of double-perovskite halides in energy devices such as photovoltaic devices ${ }^{22-24}$, photocatalysts ${ }^{25-28}$, UV detectors ${ }^{29,30}$, and solar energy storage ${ }^{31,32}$. Double-perovskite $\mathrm{R}_{2} \mathrm{CoMnO}_{6}(\mathrm{R}=\mathrm{La}, \ldots, \mathrm{Lu})$ compounds crystallize in a monoclinic double-perovskite structure $\left(P 2_{1} / n\right.$ space group), in which alternating $\mathrm{Co}^{2+}$ and $\mathrm{Mn}^{4+}$ ions are located in corner-shared octahedral $\mathrm{O}^{2-}$ environments ${ }^{33} \cdot \mathrm{Co}^{2+}$ and $\mathrm{Mn}^{4+}$ superexchange interactions result in a long-range ferromagnetic order, whose ordering temperature varies from $204 \mathrm{~K}$ for $\mathrm{La}_{2} \mathrm{CoMnO}_{6}$ to $67 \mathrm{~K}$ for $\mathrm{Er}_{2} \mathrm{CoMnO}_{6}$ as the size of the rare-earth ions decreases ${ }^{33}$. It has been known that the incomplete alteration of the $\mathrm{Co}^{2+}$ and $\mathrm{Mn}^{4+}$ ions results in a small portion of additional antiferromagnetic clusters with $\mathrm{Co}^{2+}-\mathrm{Co}^{2+}$ or $\mathrm{Mn}^{4+}-\mathrm{Mn}^{4+}$ pairs, which generate anti-sites of ionic disorders and/or antiphase boundaries ${ }^{34-37}$. Another valence state of $\mathrm{Co}^{3+}-\mathrm{Mn}^{3+}$ can be additionally formed as 
antiferromagnetic clusters ${ }^{38}$. In $\mathrm{Yb}_{2} \mathrm{CoMnO}_{6}(\mathrm{YCMO})$ and $\mathrm{Lu}_{2} \mathrm{CoMnO}_{6}$, significant distortions of $\mathrm{O}^{2-}$ octrahedra owing to the smaller size of rare-earth ions induce a magnetic frustration associated with the nearest-neighbor ferromagnetic and next-nearest-neighbor antiferromagnetic exchange interactions ${ }^{39}$. These frustrated interactions lead to up-up-down-down ( $\uparrow \downarrow \downarrow)$ spin ordering along the $c$ axis with ordering temperatures of 52 and $48 \mathrm{~K}$ for YCMO and $\mathrm{Lu}_{2} \mathrm{CoMnO}_{6}$, respectively ${ }^{40}$.

$\mathrm{Lu}_{2} \mathrm{CoMnO}_{6}$ is a double-perovskite multiferroic; it is a rare example of a multiferroic that exhibits ferromagnetic-like magnetic hysteresis with net magnetization and strong coupling to the dielectric constant and ferroelectric polarization in response to external magnetic fields ${ }^{20,41}$. The $\uparrow \uparrow \downarrow \downarrow$ spin arrangement formed by frustrated exchange interactions has been known to generate ferroelectric polarization perpendicular to the $c$ axis through the cooperative $\mathrm{O}^{2-}$ shifts via the symmetric exchange striction ${ }^{20,21,42}$. In $\mathrm{Er}_{2} \mathrm{CoMnO}_{6}$, the ferrimagnetic order activated by $\mathrm{Er}^{3+}$ moments antiparallelly aligned with ferromagnetic $\mathrm{Co}^{2+} / \mathrm{Mn}^{4+}$ sublattices results in an inversion of the magnetic hysteresis loop ${ }^{9,43}$. Moreover, the additional small portion of multiferroic phase coexisting with the ferrimagnetic phase has been presented as simultaneous metamagnetic and ferroelectric transitions ${ }^{41}$. Despite the clear demonstrations of correlated magnetic and ferroelectric properties in such multiferroics, the full hysteretic behavior in coupled magnetization and polarization and the role of magnetic rare-earth ions in multiferroicity still remain unclear.

In this study, we investigated the magnetic and magnetoelectric features of a frustrated antiferromagnet, YCMO. We have first observed the emergence of ferroelectricity perpendicular to the $c$ axis in a single crystalline YCMO. Isothermal magnetization along the $c$ axis is observed to exhibit ferromagnetic-like hysteresis with large remanent magnetization, which is strongly correlated with the isothermal dielectric constant and ferroelectric polarization perpendicular to the $c$ axis below $T_{\mathrm{N}}=52 \mathrm{~K}$. Based on the results of recent neutron diffraction experiments on YCMO, we proposed that the symmetric exchange striction between $\mathrm{Yb}^{3+}$ and $\mathrm{Co}^{2+} /$ $\mathrm{Mn}^{4+}$ moments may lead to additional ferroelectricity along the $c$ axis below $T_{\mathrm{Yb}} \approx 20 \mathrm{~K}^{44}$. We note that in typeII multiferroics, multiple ferroelectricity, in which two different sorts of ferroelectricity emerge with magnetic origins, has scarcely been observed. However, the existence of additional ferroelectric polarization was not verified. Instead, dielectric anomaly along the $c$ axis was observed. Our findings indicate distinct characteristics of the double-perovskite multiferroic and lay important groundwork for investigating materials with improved magnetoelectric functionalities.

\section{Results and discussion}

YCMO crystallizes in a monoclinic $P 2_{1} / n$ structure with lattice parameters $a=5.177 \AA, b=5.548 \AA$, and $c=7.418 \AA$ with $\beta=89.648^{\circ 41}$. The magnetic properties of YCMO were examined based on the temperature $(T)$ dependence of magnetic susceptibility, defined as magnetization $(M)$ divided by the magnetic field $(H), \chi=M / H$, measured at $H=0.2 \mathrm{~T}$ on warming after zero- $H$-cooling (ZFC) and on cooling at the same $H$ (FC). Figure $1 \mathrm{a}$ and $\mathrm{b}$ shows the anisotropic $\chi$ curves at $T=2-100 \mathrm{~K}$ for the two different orientations, $H / / c$ and $H \perp \mathrm{c}$, respectively. As $T$ decreases from $100 \mathrm{~K}$, both the ZFC and FC $\chi$ curves along the $c$ axis increase smoothly with a nearly identical shape; they show a sharp anomaly at $T_{\mathrm{N}}=52 \mathrm{~K}$, which is considered to be the emergence of the $\uparrow \uparrow \downarrow \downarrow$-type spin order $^{41,44}$. Upon further cooling below $T_{\mathrm{N}}$, the $\chi$ curves decrease considerably and begin to split around $T_{\mathrm{H}} \approx$ $37 \mathrm{~K}$, which indicates the onset of thermally hysteretic behavior similar to that of $\mathrm{Lu}_{2} \mathrm{CoMnO}_{6}{ }^{45}$. Additional and much larger splitting occurs below $T_{\mathrm{Yb}} \approx 20 \mathrm{~K}$, where the $\mathrm{Yb}^{3+}$ moments are ordered. The $\chi$ values for $H / / c$ and $H \perp \mathrm{c}$ indicate strong anisotropy, consistent with the spins primarily aligned along the $c$ axis.

In Fig. 1c, the dielectric constant $\left(\varepsilon^{\prime}\right)$ perpendicular to the $c$ axis, measured at $f=100 \mathrm{kHz}$ and $H=0,1.25,1.5$, $1.75,2.0$, and $3.0 \mathrm{~T}$ along the $c$ axis, is plotted. At zero $H, \varepsilon^{\prime}$ decreases almost linearly from $100 \mathrm{~K}$ and starts to increase near $T_{\mathrm{N}}=52 \mathrm{~K}$. It exhibits a broad peak at $T_{\mathrm{H}}$, followed by a slight slope change around $T_{\mathrm{Yb}}$. As $H$ along the $c$ axis is increased, the broad peak at $T_{\mathrm{H}}$ is progressively suppressed, and the slope change at $T_{\mathrm{Yb}}$ becomes clearer. The peak completely disappears at 3.0 T. The heat capacity divided by the temperature $(C / T)$ also shows a sharp anomaly at $T_{\mathrm{N}}$, indicating a long-range order of the $\mathrm{Co}^{2+}$ and $\mathrm{Mn}^{4+}$ moments (Fig. 1d), consistent with that observed in $\mathrm{Lu}_{2} \mathrm{CoMnO}_{6}{ }^{21}$. An additional increase in $C / T$ is found at $T_{\mathrm{Yb}}$, and a broad peak below $T_{\mathrm{Yb}}$ implies the long-range antiferromagnetic order of $\mathrm{Yb}^{3+}$ spins (Fig. 1d) ${ }^{44}$.

A previous neutron diffraction experiment on isostructural $\mathrm{Lu}_{2} \mathrm{CoMnO}_{6}$ established the magnetic structure of alternating $\mathrm{Co}^{2+}$ and $\mathrm{Mn}^{4+}$ moments as $\uparrow \uparrow \downarrow \downarrow$-type spin configurations along the $c$ axis ${ }^{46}$. The $\uparrow \uparrow \downarrow \downarrow$ spin arrangement is driven by the nearest-neighbor ferromagnetic exchange interaction frustrated with comparable next-nearest-neighbor antiferromagnetic interactions. This system is called a frustrated antiferromagnet because the magnetic moments between $\uparrow \uparrow$ and $\downarrow \downarrow$ spin layers are canceled out, similar to an A-type antiferromagnet. A recent neutron diffraction study on YCMO has shown the same $\uparrow \uparrow \downarrow \downarrow$-type arrangement of $\mathrm{Co}^{2+}$ and $\mathrm{Mn}^{4+}$ moments ${ }^{41}$. Figure 2a depicts the two types of frustrated antiferromagnetic domains of YCMO projected onto the $b c$ plane. In the $\uparrow \uparrow \downarrow \downarrow$ spin configuration as one type of domain, the parallel spin pairs of $\mathrm{Co}^{2+}$ and $\mathrm{Mn}^{4+}$ ions tend to contract, while the antiparallel spin pairs tend to expand, based on the exchange striction, which prefers ferromagnetic nearest-neighbor coupling. As a result, the contraction and expansion of the spin pairs distort between $\mathrm{O}^{2-}$ ions with cooperative displacements to the left and generate ferroelectric polarization $(P)$ along the $b$ axis, $+P$. For the other type of domains with the $\uparrow \downarrow \downarrow \uparrow$ spin configuration, the exchange striction induces displacements of $\mathrm{O}^{2-}$ ions to the right and thus produces $-P$.

The full magnetic curve for $H / / c$ was recorded at $3 \mathrm{~K}$ after ZFC up to $\pm 6 \mathrm{~T}$, as shown in Fig. $2 \mathrm{~b}$. The $\uparrow \uparrow \downarrow \downarrow$-type spin order exhibits a ferromagnetic-like hysteresis loop with remanent $M, M_{\mathrm{r}}=5.1 \mu_{\mathrm{B}} /$ f.u., and magnetic coercive field, $H_{\mathrm{c}}=1.4 \mathrm{~T}$. The initial curve increases slowly and shows a sudden jump at $\sim 3.8 \mathrm{~T}$, indicating a change in the spin state from $\uparrow \uparrow \downarrow \downarrow$ to $\uparrow \uparrow \uparrow \uparrow$. Above this value, $M$ increases linearly without saturation. The value of $M$ at $6 \mathrm{~T}$ is found to be $\sim 6.5 \mu_{\mathrm{B}} /$ f.u., ascribed to the magnetic moments of $\mathrm{Yb}^{3+}$ ions in addition to the $M$ value of 6 $\mu_{\mathrm{B}}$ for the $\mathrm{Co}^{2+}(S=3 / 2)$ and $\mathrm{Mn}^{4+}(S=3 / 2)$ moments in a formula unit. Upon decreasing $H$ from $6 \mathrm{~T}, M$ reduces 

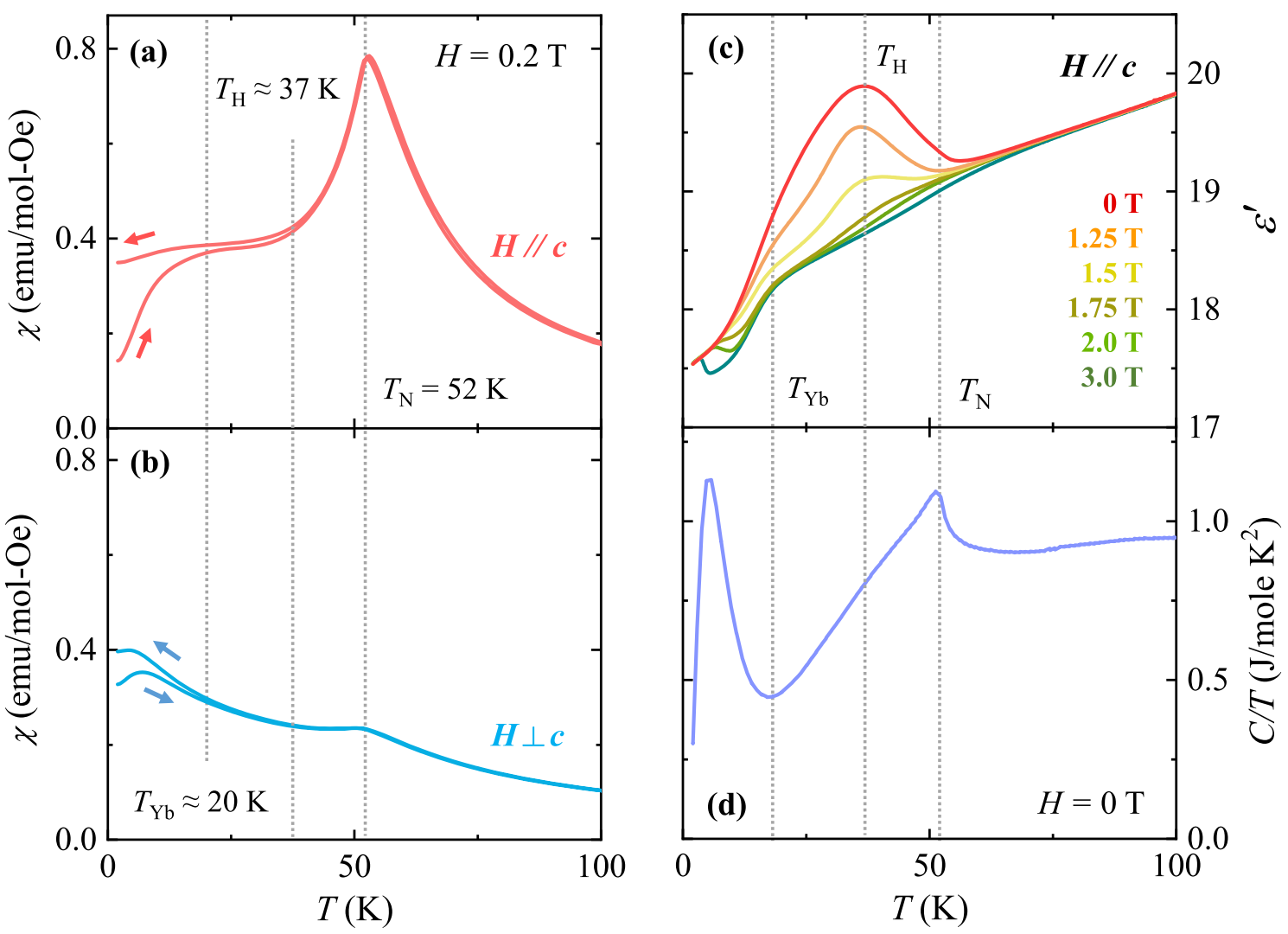

Figure 1. Temperature-dependent physical properties of $\mathrm{Yb}_{2} \mathrm{CoMnO}_{6}(\mathrm{YCMO})$. (a) and (b) Temperature dependence of magnetic susceptibility, $\chi=M / H$, of the YCMO crystal at $H=0.2$ T upon warming after zeromagnetic-field cooling and upon cooling in the same field along and perpendicular to the $c$ axis, respectively. The vertical dotted lines represent the ordering temperature forming $\uparrow \uparrow \downarrow \downarrow$-type spin state $\left(T_{\mathrm{N}}=52 \mathrm{~K}\right)$, onset temperature of magnetic hysteresis $\left(T_{\mathrm{H}} \approx 37 \mathrm{~K}\right)$, and ordering temperature of $\mathrm{Yb}^{3+}$ moments $\left(T_{\mathrm{Yb}} \approx 20 \mathrm{~K}\right)$. (c) Temperature dependence of dielectric constant $\left(\varepsilon^{\prime}\right)$, measured perpendicular to the $c$ axis at $H=0,1.25,1.5$, $1.75,2.0,3.0 \mathrm{~T}$ along the $c$ axis. (d) Temperature dependence of specific heat divided by the temperature, $C / T$, measured at $H=0 \mathrm{~T}$.

progressively until it shows an abrupt decrease at approximately $-0.8 \mathrm{~T}$ with the formation of the $\uparrow \uparrow \downarrow \downarrow$-type magnetic domains. As $H$ is further decreased, another step arises at $-3.8 \mathrm{~T}$ for the change in the spin state to $\downarrow \downarrow \downarrow \downarrow$. The antisymmetric shape of the loop is accomplished by sweeping $H$ in the other direction.

The magnetoelectrically hysteretic behavior of YCMO was examined through the isothermal ferroelectric $P$, obtained by integrating the pyroelectric current density measured after poling in an electric field $(E=100 \mathrm{~V}$ or $E=2.3 \mathrm{kV} / \mathrm{cm}$ ) perpendicular to the $c$ axis and $H$ up to $\pm 6 \mathrm{~T}$ along the $c$ axis. In Fig. 2c, P was measured at $3 \mathrm{~K}$ by sweeping $H$ without removing $E$ after poling. The magnitude of $P$ at $0 \mathrm{~T}$ was $4.3 \mu \mathrm{C} / \mathrm{m}^{2}$. $P$ in the initial curve disappears at 3.8 T, consistent with the change in the spin state from $\uparrow \uparrow \downarrow \downarrow$ to $\uparrow \uparrow \uparrow \uparrow$. By decreasing $H$ from $6 \mathrm{~T}, P$ is still zero until it shows a sudden jump at $-0.8 \mathrm{~T}$ owing to the almost recovery of the $\uparrow \uparrow \downarrow \downarrow$ state. $P$ is reduced to zero again at $-3.8 \mathrm{~T}$ for the change in the spin state to $\downarrow \downarrow \downarrow \downarrow$. A similar hysteretic behavior was observed for the other directions of $H$. In Fig. $2 \mathrm{~d}, \mathrm{P}$ was measured by sweeping $H$ at $E=0 \mathrm{~V}$ after poling. The $P$ value at $0 \mathrm{~T}$ was reduced to $1.6 \mu \mathrm{C} / \mathrm{m}^{2}$, which suggests the incorporation of a considerable portion of the $\uparrow \downarrow \downarrow \uparrow$-type antiferromagnetic domains with the opposite sign of $P$. The overall value of $P$ was diminished, but a similar hysteretic behavior was detected. In addition, peak anomalies were observed when $P$ was reduced to zero and recovered. In Fig. 2e, the magnetodielectric (MD) effect, described by the variation in $\varepsilon^{\prime}$ by applying $H$ and defined as MD $(\%)=\frac{\varepsilon^{\prime}(H)-\varepsilon^{\prime}(0 \mathrm{~T})}{\varepsilon^{\prime}(0 \mathrm{~T})} \times 100$, was measured at $f=100 \mathrm{kHz}$ and $T=3 \mathrm{~K}$ perpendicular to the $c$ axis up to $\pm 6 \mathrm{~T}$ along the $c$ axis. $\varepsilon^{\prime}$ reveals a hysteretic behavior similar to that of $P$, which reflects the ferroelectric domain motion.

To examine the behavior of full magnetoelectric hysteresis in detail, the $T$ evolution of various physical properties was measured. Figure 3 shows a comparison among isothermal $P$ (measured at $E=100 \mathrm{~V}$ ) and MD effect perpendicular to the $c$ axis, and $M$ along the $c$ axis at $H$ up to $\pm 6 \mathrm{~T}$ and $T=5,10,20,30$, and $40 \mathrm{~K}$. As $T$ is increased, the hysteretic behavior is gradually suppressed. At $5 \mathrm{~K}$, the area within the magnetic hysteresis loop is reduced (Fig. 3a), accompanied by a large reduction in $M_{\mathrm{r}}=1.73 \mu_{\mathrm{B}} /$ f.u. and $H_{\mathrm{c}}=0.78 \mathrm{~T}$, in comparison with $M$ at $3 \mathrm{~K}$ (Fig. 2b). Owing to the narrowed hysteresis loop, double-step variations in $M$ occur at $H=0.24$ and $-3.07 \mathrm{~T}$ upon sweeping $H$ from $6 \mathrm{~T}$. At $10 \mathrm{~K}$, the magnetic hysteresis narrows further with $M_{\mathrm{r}}=0.23 \mu_{\mathrm{B}} /$ f.u. and $H_{\mathrm{c}}=0.17 \mathrm{~T}$ (Fig. 3b). As $T$ increases further, the area of the magnetic hysteresis loop of $M$ rapidly shrinks, and $M_{r}$ and $H_{c}$ are nearly suppressed. At $40 \mathrm{~K}$, the magnetic hysteresis in $M$ almost disappears, while the double-step transitions remain (Fig. 3e). As shown in Fig. 3f, P at $5 \mathrm{~K}$ shows a hysteretic behavior similar to $M$ following the 

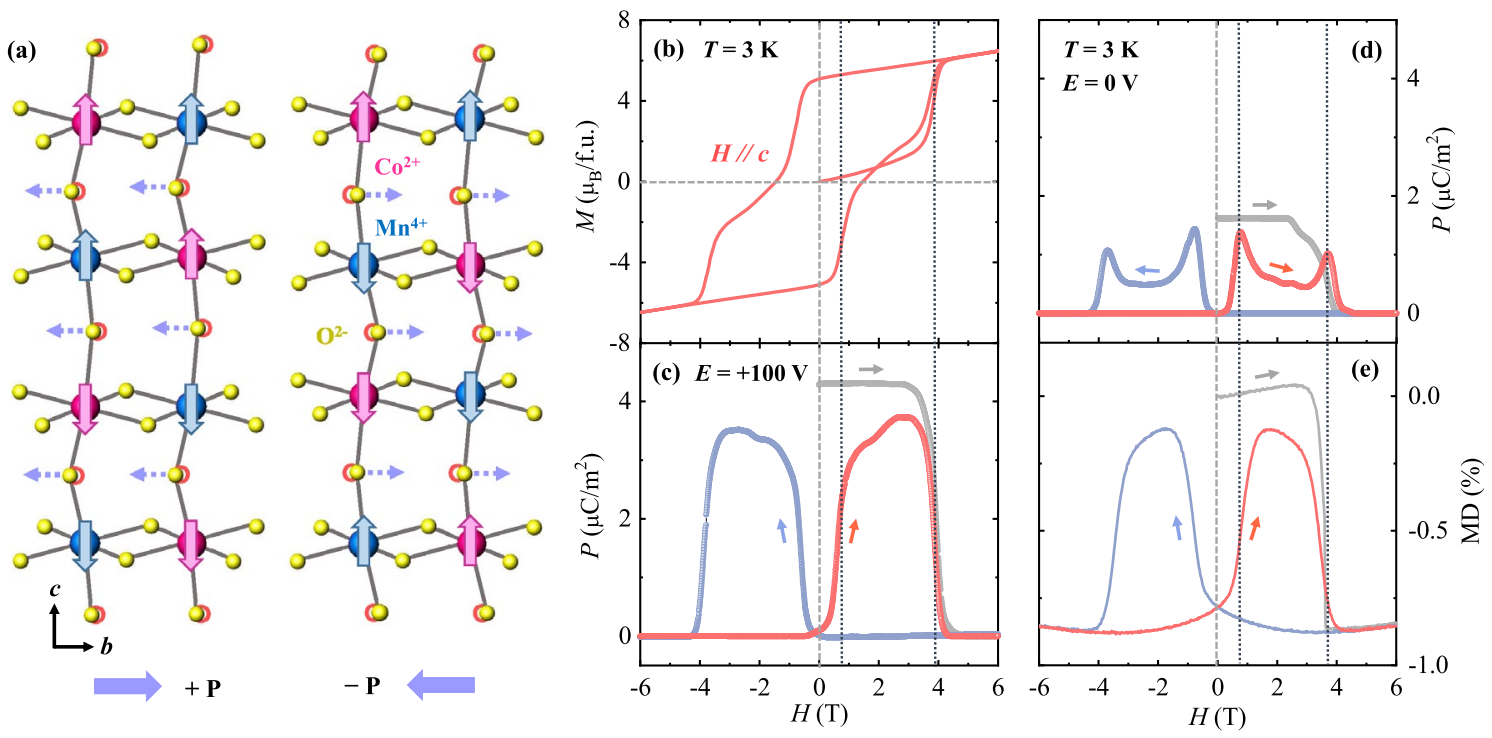

Figure 2. Origin of ferroelectricity in frustrated antiferromagnetic domains and magnetoelectrically hysteretic behavior. (a) Two types of frustrated antiferromagnetic domains. The purple dotted arrows denote the shifts in $\mathrm{O}^{2-}$ ions due to the exchange striction between parallel and antiparallel neighboring spins. (b) Isothermal magnetization of the YCMO crystal measured at $T=3 \mathrm{~K}$ in an $H$ range of $\pm 6 \mathrm{~T}$ along the $c$ axis. (c) and (d) Isothermal polarization perpendicular to the $c$ axis at $T=3 \mathrm{~K}$ and $H$ along the $c$ axis, measured with $E=100$ and $0 \mathrm{~V}$, respectively. (e) Magnetodielectric (MD) effect defined as $\mathrm{MD}(\%)=\frac{\varepsilon^{\prime}(H)-\varepsilon^{\prime}(0 \mathrm{~T})}{\varepsilon^{\prime}(0 \mathrm{~T})} \times 100$ perpendicular to the $c$ axis for $H$ up to $\pm 6 \mathrm{~T}$ along the $c$ axis.

appearance and disappearance of ferroelectricity at the double steps, which suggests a strong correlation between the magnetic and ferroelectric properties. As $T$ increases further, the hysteretic behavior of $P$ is reduced, but the magnitude of $P$ is considerably enhanced up to $20 \mathrm{~K}$ (Fig. $3 \mathrm{~g}$ and h). The maximum $P$ values at 10 and $20 \mathrm{~K}$ were found to be 10.71 and $13.74 \mu \mathrm{C} / \mathrm{m}^{2}$, respectively. Above $20 \mathrm{~K}$, the magnitude of $P$ decreases with further reduction in the hysteretic behavior (Fig. 3i and j). The MD also tends to behave akin to $P$, which reflects the $H$-driven variations in ferroelectricity (Fig. $3 \mathrm{k}-\mathrm{o}$ ). The variation in MD is progressively enhanced as $T$ increases. At 30 and $40 \mathrm{~K}$, the maximum MD values were observed as $-6.68 \%$ and $-5.26 \%$, respectively, at $6 \mathrm{~T}$. These large variations arise at the $T$ regime close to $T_{\mathrm{H}} \approx 37 \mathrm{~K}$, at which the broad peak of $\varepsilon^{\prime}$ occurs (Fig. $1 \mathrm{c}$ ). A recent X-ray photon correlation spectroscopy study on $\mathrm{Lu}_{2} \mathrm{CoMnO}_{6}$ clarified that the $\uparrow \uparrow \downarrow \downarrow$-type arrangement, which is slightly incommensurate (ICM) with $\boldsymbol{k}=(0.0223(8), 0.0098(7), 0.5)^{46}$, emerges at $T_{\mathrm{N}}=48 \mathrm{~K}$ and commensurate $(\mathrm{CM})$ spin order corresponding to $\boldsymbol{k}=(0,0,0.5)$ arises below $T_{\mathrm{H}}=30 \mathrm{~K}$, while the ICM order still remains ${ }^{47}$. This suggests that the strong magnetic hysteresis originates from the simultaneous presence of the ICM and CM orders. The comprehensive behavior of strong magnetoelectric hysteresis in YCMO is demonstrated in the $H-T$ phase diagram constructed from $T$ dependence of $M$, and $H$ dependence of $M$ and $P$, as shown in Fig. 4 . The data points for the phase boundaries were attained from the maximum slopes of $H$ dependence of $M$ and $P$. The onset of magnetoelectric hysteresis emerges at $T_{\mathrm{H}} \approx 37 \mathrm{~K}$, below which the hysteretic regime expands continuously. The red regime in which the corresponding magnetic state relies on magnetic hysteresis is clearly presented. The $P$ is zero in the green colored region, corresponding to $\uparrow \uparrow \uparrow \uparrow$ or $\downarrow \downarrow \downarrow \downarrow \mathrm{Co}^{2+} / \mathrm{Mn}^{4+}$ spin configuration due to the large magnetoelectric hysteresis at low $T$ regime.

In a recent neutron diffraction study on YCMO, in addition to the $\uparrow \uparrow \downarrow \downarrow$-type arrangement of $\mathrm{Co}^{2+}$ and $\mathrm{Mn}^{4+}$ moments, the ordering of $\mathrm{Yb}^{3+}$ spins has been observed ${ }^{41}$. From the spin structure attained from this previously investigated neutron diffraction, we propose schematic spin configurations forming four types of magnetoelectric domains and possible activation of the symmetric exchange striction between $\mathrm{Yb}^{3+}$ and $\mathrm{Co}^{2+} / \mathrm{Mn}^{4+}$ moments, which generates an additional ferroelectric $P$ along the $c$ axis, as shown in Fig. 5. All spin configurations are established within the magnetic $P_{a} 2_{1}$ symmetry, consistent with that of $\mathrm{Lu}_{2} \mathrm{CoMnO}_{6}$. In such a symmetry, $\mathrm{Yb}^{3+}$ spins are distinguished as two different sites. For $\mathrm{Yb} 1$ sites, $\mathrm{Yb}^{3+}$ spins turn out to be strongly disordered because of the formation of strong internal fields from the neighboring ferromagnetic arrangement of $\mathrm{Co}^{2+} / \mathrm{Mn}^{4+}$ spins between the upper and lower layers, which inhibits the antiferromagnetic coupling of $\mathrm{Yb}^{3+} \mathrm{spins}^{41}$. By contrast, at the $\mathrm{Yb} 2$ sites, the cancelation of the internal fields from neighboring antiferromagnetic arrangement enables the $\mathrm{Yb}^{3+}$ spins to be ordered ${ }^{41}$. In Fig. $5 \mathrm{a}$, the $\mathrm{Yb}^{3+}$ moments in the $\mathrm{Yb} 2$ sites are ordered antiferromagnetically aside from the $\uparrow \uparrow \downarrow \downarrow$ spin arrangement of the $\mathrm{Co}^{2+}$ and $\mathrm{Mn}^{4+}$ ions; they also prefer to align antiferromagnetically to the neighboring $\mathrm{Co}^{2+} / \mathrm{Mn}^{4+}$ moments. In such configuration, the down $(\downarrow) \mathrm{Yb}^{3+}$ spin tends to shift to the upward direction and the up $(\uparrow) \mathrm{Yb}^{3+}$ spin also shifts to the upward direction owing to the exchange striction that prefers the antiparallel alignment between $\mathrm{Yb}^{3+}$ and $\mathrm{Co}^{2+} / \mathrm{Mn}^{4+}$ spins. Thus, the net ferroelectric $P$ is produced along the $c$ axis. Similarly, oppositely aligned ferroelectric $P$ occurs in the spin configuration, as shown in Fig. 5b. The other two types of magnetoelectric domains are depicted in Fig. $5 \mathrm{c}$ and d. Accompanied by the $\uparrow \downarrow \downarrow \uparrow$ spin arrangement of the $\mathrm{Co}^{2+}$ and $\mathrm{Mn}^{4+}$ ions, the antiferromagnetic order of $\mathrm{Yb}^{3+}$ spins also leads to $+P$ and $-P$ along 

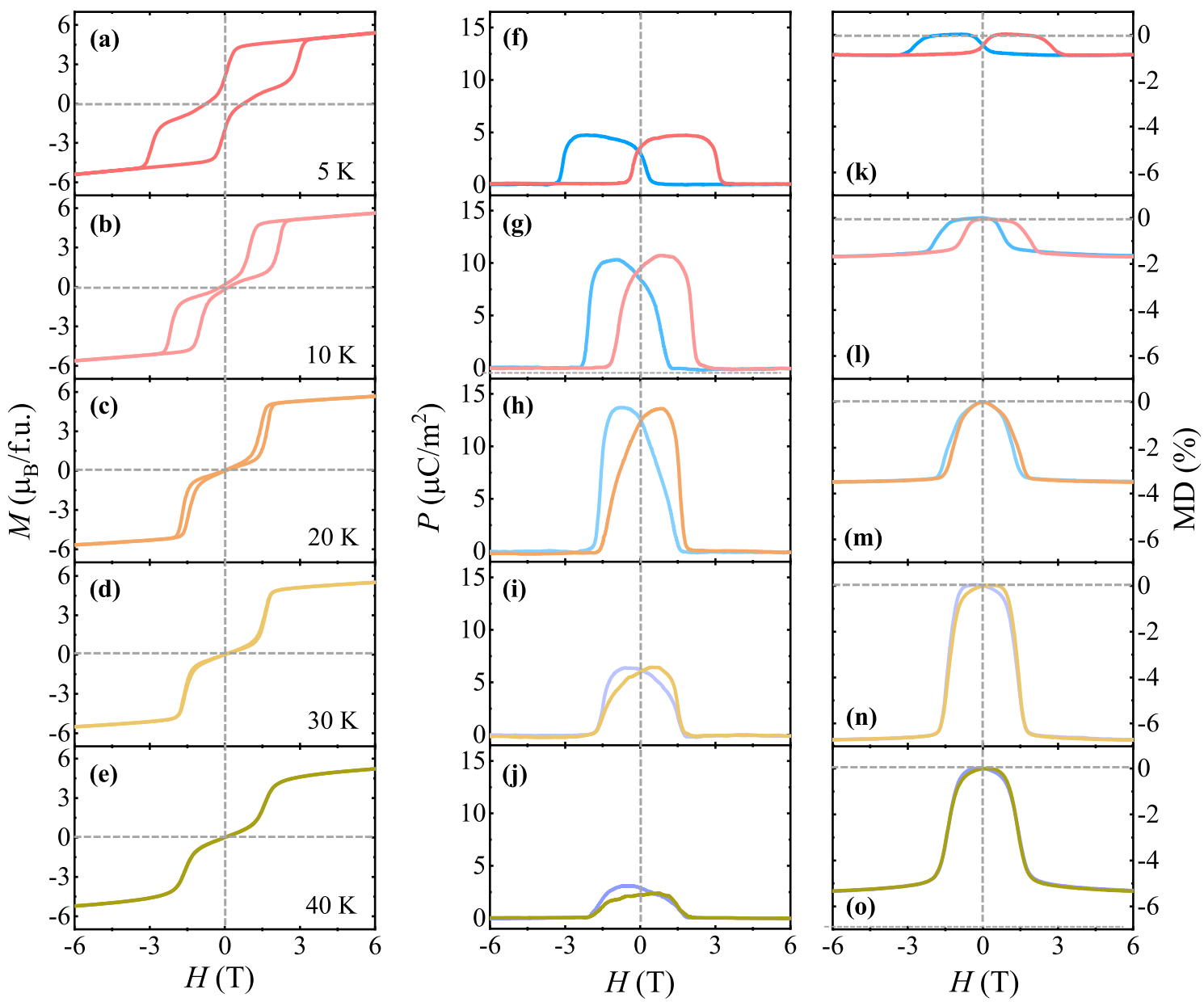

Figure 3. Temperature evolution of magnetic and ferroelectric properties. (a)-(e) Isothermal magnetization at $H$ up to $\pm 6 \mathrm{~T}$ along the $c$ axis and $T=5,10,20,30$, and $40 \mathrm{~K}$. (f) $-(\mathbf{j})$ Ferroelectric polarization perpendicular to the $c$ axis at $H$ up to $\pm 6 \mathrm{~T}$ along the $c$ axis and $T=5,10,20,30$, and $40 \mathrm{~K}$, obtained by integrating the magnetoelectric current measured and changing $H$ at the rate of $0.01 \mathrm{~T} / \mathrm{s}$ up to $\pm 6 \mathrm{~T}$ after poling at $E=2.3 \mathrm{kV} /$ $\mathrm{cm}$. (k)-(o) Magnetodielectric (MD) effect defined as $\mathrm{MD}(\%)=\frac{\varepsilon^{\prime}(H)-\varepsilon^{\prime}(0 \mathrm{~T})}{\varepsilon^{\prime}(0 \mathrm{~T})} \times 100$ perpendicular to the $c$ axis for $H$ up to $\pm 6 \mathrm{~T}$ along the $c$ axis at $T=5,10,20,30$, and $40 \mathrm{~K}$.

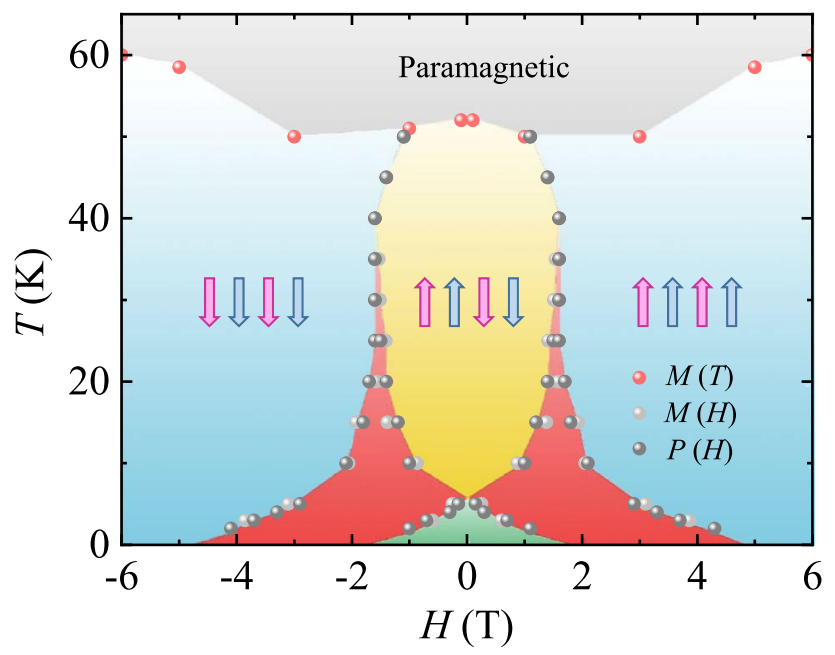

Figure 4. Phase diagram. The $H$-T phase diagram of the YCMO single crystal for $H$ along the $c$ axis, constructed from $T$ dependence of $M$, and $H$ dependence of $M$ and $P$. In the red region, magnetic structure depends on the hysteresis. 

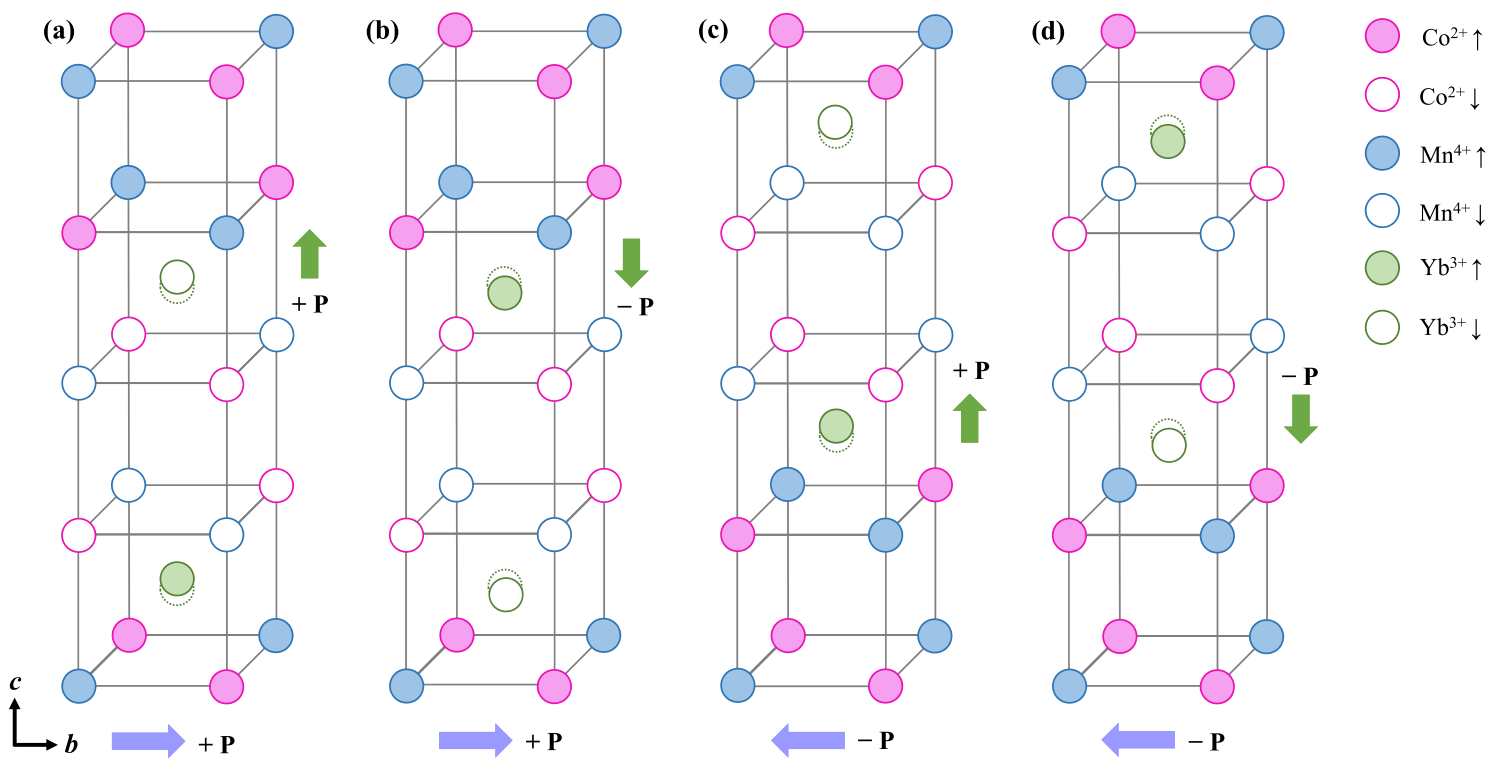

Figure 5. Proposed magnetoelectric domains of YCMO. Four types of magnetoelectric domains constructed from recent neutron diffraction experiments. The pink, blue, and green circles denote $\mathrm{Co}^{2+}, \mathrm{Mn}^{4+}$, and $\mathrm{Yb}^{3+}$ ions, respectively. The filled and open circles represent up and down spins, respectively, and the green dotted circle indicates the original position of the $\mathrm{Yb}^{3+}$ ions. In addition to $P$ along the $b$ axis, the exchange striction between $\mathrm{Yb}^{3+}$ and $\mathrm{Co}^{2+} / \mathrm{Mn}^{4+}$ magnetic moments induces $P$ along the $c$ axis. The four types of magnetoelectric domains are distinguished as $\left(P_{b}, P_{c}\right)=(+,+),(+,-),(-,+)$, and $(-,-)$.

the $c$ axis, respectively. As a result, four different types of magnetoelectric domains form depending on both $P$ directions, i.e., $\left(P_{b}, P_{c}\right)=(+,+),(+,-),(-,+)$, and $(-,-)$.

To evaluate the potential occurrence of additional ferroelectricity along the $c$ axis, we measured the electric properties. Contrary to the overall negative MD perpendicular to the $c$ axis (Figs. 2e and $3 \mathrm{k}-\mathrm{o}$ ), the MD effect along the $c$ axis with $H$ up to $\pm 6 \mathrm{~T}$ along the $c$ axis appears to be positive, and the magnitude of MD is largely reduced, as shown in Fig. 6 . At $3 \mathrm{~K}$, the initial curve exhibits a step-like jump in accordance with the spin-state variation from $\uparrow \uparrow \downarrow \downarrow$ to $\uparrow \uparrow \uparrow \uparrow$ (Fig. 6a). Following the magnetically hysteretic behavior, a rapid decrease in MD is observed at a negative value of $H$. A further decrease in $H$ leads to a sudden jump, indicating the magnetic transition to the $\downarrow \downarrow \downarrow \downarrow$ state. As $T$ increases further, the magnitude of MD is continually reduced, which is different from the $T$ evolution of the MD effect perpendicular to the $c$ axis (Fig. $6 \mathrm{~b}-\mathrm{d}$ ). Despite the observed positive MD effect with a strong dielectric anomaly, the presence of the proposed additional ferroelectricity has not been clearly proven because no measurable electric $P$ was detected within the accuracy of our pyroelectric current measurement. Note that our measurement is highly sensitive to the $P$ magnitude, as shown in the measured $P$ perpendicular to the $c$ axis (Figs. $2 \mathrm{c}, \mathrm{d}$, and $3 \mathrm{f}-\mathrm{j}$ ).

\section{Conclusion}

In summary, we explored the anisotropic magnetoelectric properties of the frustrated antiferromagnet YCMO. Ferroelectric polarization was found to occur perpendicular to the $c$ axis, originating from the exchange strictive shifts of $\mathrm{O}^{2-}$ ions of the $\uparrow \uparrow \downarrow \downarrow$-type $\mathrm{Co}^{2+} / \mathrm{Mn}^{4+}$ spin order along the $c$ axis. The magnetoelectric hysteresis observed below $T_{\mathrm{H}} \approx 37 \mathrm{~K}$ revealed an interesting correlation between the magnetic and ferroelectric properties. We also proposed that the magnetic order of $\mathrm{Yb}^{3+}$ moments below $T_{\mathrm{Yb}} \approx 20 \mathrm{~K}$ could lead to an additional ferroelectric polarization along the $c$ axis with the formation of four different types of magnetoelectric domains. Our findings provide insights into fundamental magnetic and magnetoelectric interactions in the frustrated antiferromagnets of the double-perovskite family, inspiring the discovery of new compounds for functional magnetoelectric applications.

\section{Methods}

Single crystals of double-perovskite YCMO were synthesized by the conventional flux method using $\mathrm{Bi}_{2} \mathrm{O}_{3}$ as a flux ${ }^{40}$. A polycrystalline specimen was first prepared by a solid-state reaction. High-purity powders of $\mathrm{Yb}_{2} \mathrm{O}_{3}$, $\mathrm{Co}_{3} \mathrm{O}_{4}$, and $\mathrm{MnO}_{2}$ were mixed in a stoichiometric ratio and ground in a mortar, followed by pelletizing and calcining at $1000^{\circ} \mathrm{C}$ for $12 \mathrm{~h}$ in a furnace. The calcined pellets were reground and sintered at $1100^{\circ} \mathrm{C}$ for $24 \mathrm{~h}$. The same sintering procedure was repeated at $1200^{\circ} \mathrm{C}$ for $48 \mathrm{~h}$. A mixture of polycrystalline powder and $\mathrm{Bi}_{2} \mathrm{O}_{3}$ flux in a $1: 12$ ratio was heated to $1300^{\circ} \mathrm{C}$ and melted in a Pt crucible. It was then slowly cooled to $850{ }^{\circ} \mathrm{C}$ at a rate of $1.5^{\circ} \mathrm{C} / \mathrm{h}$ and then cooled further to room temperature while the furnace was turned off.

The temperature and magnetic-field dependences of the DC magnetizations were measured using a vibrating sample magnetometer (VSM) at $T=2-100 \mathrm{~K}$ and $H=-9-9 \mathrm{~T}$ in a physical properties measurement system (PPMS, Quantum Design, Inc.). The specific heat was measured using the standard relaxation method in the PPMS. The temperature and magnetic-field dependences of the dielectric constant were measured at $f=100 \mathrm{kHz}$ 


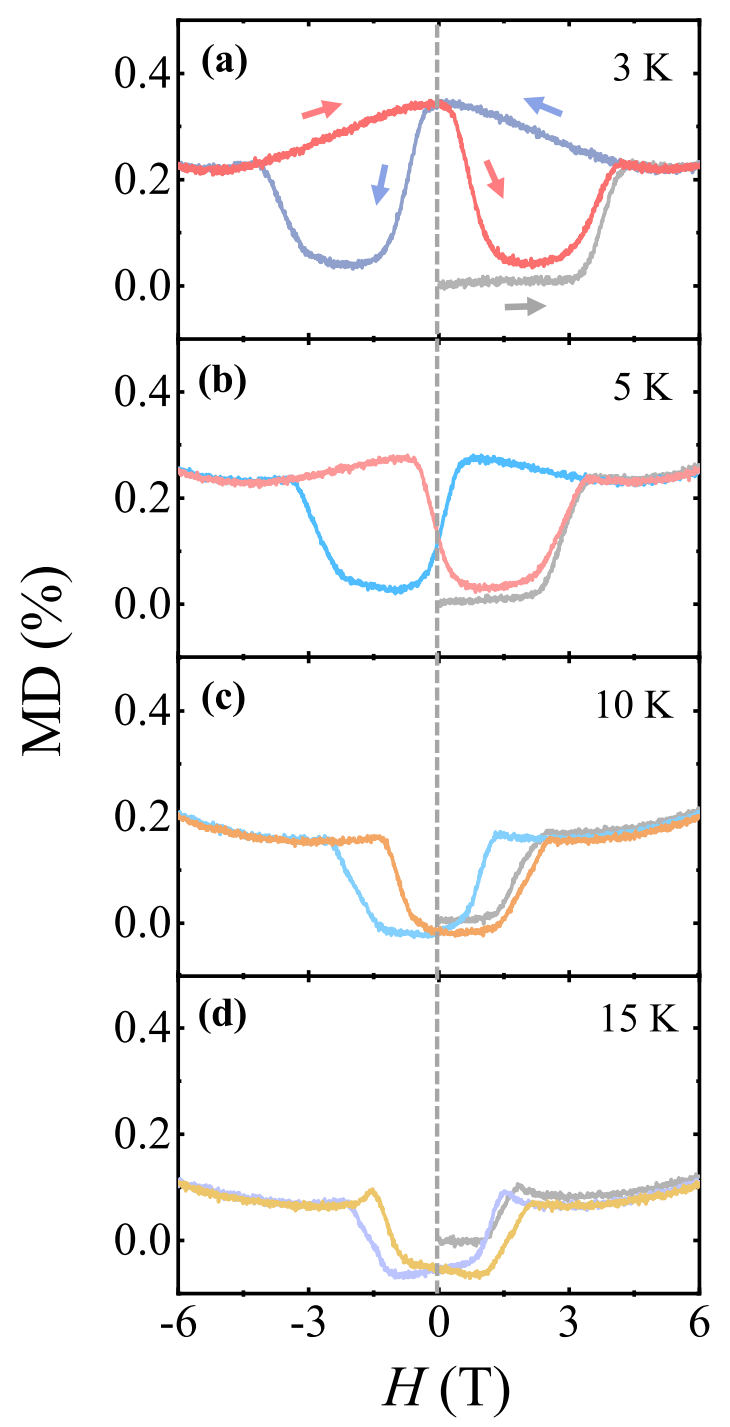

Figure 6. Temperature evolution of magnetodielectric effect along the $c$ axis. (a)-(d) Magnetodielectric (MD) effect, $\mathrm{MD}(\%)=\frac{\varepsilon^{\prime}(H)-\varepsilon^{\prime}(0 \mathrm{~T})}{\varepsilon^{\prime}(0 \mathrm{~T})} \times 100$, along the $c$ axis for $H$ up to $\pm 6 \mathrm{~T}$ along the $c$ axis at $T=3,5,10$, and $20 \mathrm{~K}$.

using an LCR meter (E4980, Agilent). The temperature and magnetic-field dependences of electric polarization were obtained by integrating pyro- and magneto-electric currents, respectively, measured after poling in a static electric field, $E=2.3 \mathrm{kV} / \mathrm{cm}$.

Received: 18 September 2021; Accepted: 29 November 2021

Published online: 10 December 2021

\section{References}

1. AJ Hearmon 2012 Electric field control of the magnetic chiralities in ferroaxial multiferroic $\operatorname{RbFe}\left(\mathrm{MoO}_{4}\right)_{2}$ Phys. Rev. Lett. 108 237201 https://doi.org/10.1103/PhysRevLett.108.237201

2. V Kocsis 2019 Magnetization-polarization cross-control near room temperature in hexaferrite single crystals Nat. Commun. 10 1247 https://doi.org/10.1038/s41467-019-09205-x

3. YJ Choi CL Zhang N Lee SW Cheong 2010 Cross-control of magnetization and polarization by electric and magnetic fields with competing multiferroic and weak-ferromagnetic phases Phys. Rev. Lett. 105097201 https://doi.org/10.1103/PhysRevLett.105. 097201

4. N Lee 2013 Giant tunability of ferroelectric polarization in $\mathrm{GdMn}_{2} \mathrm{O}_{5}$ Phys. Rev. Lett. $110137203 \mathrm{https} / /$ doi.org/10.1103/PhysR evLett.110.137203

5. S-W Cheong M Mostovoy 2007 Multiferroics: A magnetic twist for ferroelectricity Nat. Mater. 61320 https://doi.org/10.1038/ nmat 1804

6. M Fiebig T Lottermoser D Meier M Trassin 2016 The evolution of multiferroics Nat. Rev. Mater. 116046 https://doi.org/10.1038/ natrevmats.2016.46

7. AB Sushkov 2014 Spectral origin of the colossal magnetodielectric effect in multiferroic $\mathrm{DyMn}_{2} \mathrm{O}_{5}$ Phys. Rev. B 90054417 https:// doi.org/10.1103/PhysRevB.90.054417

8. G Cao 2014 Novel magnetism of $\operatorname{Ir}^{5+}\left(5 d^{4}\right)$ ions in the double perovskite $\mathrm{Sr}_{2} \mathrm{YIrO}_{6}$ Phys. Rev. Lett. 112056402 https://doi.org/10. 1103/PhysRevLett.112.056402 
9. MK Kim 2019 Strong magnetoelectric coupling in mixed ferrimagnetic-multiferroic phases of a double perovskite Sci. Rep. 9 5456 https://doi.org/10.1038/s41598-019-41990-9

10. X Ding 2019 Magnetic properties of double perovskite $\mathrm{Ln}_{2} \mathrm{CoIrO}_{6}(\mathrm{Ln}=\mathrm{Eu}, \mathrm{Tb}, \mathrm{Ho})$ : Hetero-tri-spin 3d-5d-4f systems Phys. Rev. B 99014438 https://doi.org/10.1103/PhysRevB.99.014438

11. R Pradheesh HS Nair V Sankaranarayanan K Sethupathi 2012 Exchange bias and memory effect in double perovskite $\mathrm{Sr}_{2} \mathrm{FeCoO}_{6}$ Appl. Phys. Lett. 101142401 https://doi.org/10.1063/1.4756792

12. W Liu 2014 Griffiths phase, spin-phonon coupling, and exchange bias effect in double perovskite $\mathrm{Pr}_{2} \mathrm{CoMnO}_{6}$ J. Appl. Phys. 116 193901 https://doi.org/10.1063/1.4902078

13. JK Murthy A Venimadhav 2017 4f-3d exchange coupling induced exchange bias and field induced Hopkinson peak effects in $\mathrm{Gd}_{2} \mathrm{CoMnO}_{6}$ J. Alloys Compd. 719341346 https://doi.org/10.1016/j.jallcom.2017.05.203

14. JY Moon 2018 Anisotropic magnetic properties and giant rotating magnetocaloric effect in double-perovskite $\mathrm{Tb}_{2} \mathrm{CoMnO}_{6} \mathrm{Phys}$ Rev. B 98174424 https://doi.org/10.1103/PhysRevB.98.174424

15. JY Moon MK Kim YJ Choi N Lee 2017 Giant anisotropic magnetocaloric effect in double-perovskite $\mathrm{Gd}_{2} \mathrm{CoMnO}_{6}$ single crystals Sci. Rep. 716099 https://doi.org/10.1038/s41598-017-16416-z

16. C Ganeshraj R Pradheesh PN Santhosh 2012 Structural, magnetic, transport and magnetocaloric properties of metamagnetic $\mathrm{DyMn}_{0.5} \mathrm{Co}_{0.5} \mathrm{O}_{3}$ J. Appl. Phys. 111 07A914 https://doi.org/10.1063/1.3672067

17. M Balli P Fournier S Jandl KD Truong MM Gospodinov 2014 Analysis of the phase transition and magneto-thermal properties in $\mathrm{La}_{2} \mathrm{CoMnO}_{6}$ single crystals J. Appl. Phys. 116073907 https://doi.org/10.1063/1.4893721

18. N Terada 2015 Ferroelectricity induced by ferriaxial crystal rotation and spin helicity in a B-site-ordered double-perovskite multiferroic $\mathrm{In}_{2} \mathrm{NiMnO}_{6}$ Phys. Rev. B 91104413 https://doi.org/10.1103/PhysRevB.91.104413

19. G Sharma J Saha SD Kaushik V Siruguri S Patnaik 2013 Magnetism driven ferroelectricity above liquid nitrogen temperature in $\mathrm{Y}_{2} \mathrm{CoMnO}_{6}$ Appl. Phys. Lett. 103012903 https://doi.org/10.1063/1.4812728

20. S Chikara 2016 Electric polarization observed in single crystals of multiferroic $\mathrm{Lu}_{2} \mathrm{MnCoO}_{6}$ Phys. Rev. B 93180405 https://doi. org/10.1103/PhysRevB.93.180405

21. N Lee 2014 Strong ferromagnetic-dielectric coupling in multiferroic $\mathrm{Lu}_{2} \mathrm{CoMnO}_{6}$ single crystals Appl. Phys. Lett. 104112907 https://doi.org/10.1063/1.4869479

22. AH Slavney T Hu AM Lindenberg HI Karunadasa 2016 A bismuth-halide double perovskite with long carrier recombination lifetime for photovoltaic applications J. Am. Chem. Soc. 13821382141 https://doi.org/10.1021/jacs.5b13294

23. L Schade 2019 Structural and optical properties of $\mathrm{Cs}_{2} \mathrm{AgBiBr}_{6}$ double perovskite ACS Energy Lett. 4299305 https://doi.org/10. 1021/acsenergylett.8b02090

24. G Longo 2020 Understanding the performance-limiting factors of $\mathrm{Cs}_{2} \mathrm{AgBiBr}_{6}$ double-perovskite solar cells ACS Energy Lett. 5 22002207 https://doi.org/10.1021/acsenergylett.0c01020

25. Z Zhang 2019 Stable and highly efficient photocatalysis with lead-free double-perovskite of $\mathrm{Cs}_{2} \mathrm{AgBiBr}_{6}$ Angew. Chem. Int. Ed. 5872637267 https://doi.org/10.1002/anie.201900658

26. T Wang D Yue X Li Y Zhao 2020 Lead-free double perovskite $\mathrm{Cs}_{2} \mathrm{AgBiBr}_{6} / \mathrm{RGO}$ composite for efficient visible light photocatalytic $\mathrm{H}_{2}$ evolution Appl. Catal. B 268118399 https://doi.org/10.1016/j.apcatb.2019.118399

27. L Zhou Y-F Xu B-X Chen D-B Kuang C-Y Su 2018 Synthesis and photocatalytic application of stable lead-free $\mathrm{Cs}_{2} \mathrm{AgBiBr}_{6}$ per-

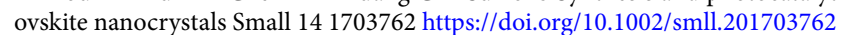

28. Z Liu 2021 Synthesis of lead-free $\mathrm{Cs}_{2} \mathrm{AgBiX}_{6}\left(\mathrm{X}=\mathrm{Cl}, \mathrm{Br}\right.$, I) double perovskite nanoplatelets and their application in $\mathrm{CO}_{2}$ photocatalytic reduction Nano Lett. 2116201627 https://doi.org/10.1021/acs.nanolett.0c04148

29. M Wang P Zeng Z Wang M Liu 2020 Vapor-deposited $\mathrm{Cs}_{2} \mathrm{AgBiCl}_{6}$ double perovskite films toward highly selective and stable ultraviolet photodetector Adv. Sci. 71903662 https://doi.org/10.1002/advs.201903662

30. J Luo $2018 \mathrm{Cs}_{2} \mathrm{AgInCl}_{6}$ double perovskite single crystals: Parity forbidden transitions and their application for sensitive and fast UV photodetectors ACS Photon. 5398405 https://doi.org/10.1021/acsphotonics.7b00837

31. M Ghasemi 2020 Dual-ion-diffusion induced degradation in lead-free $\mathrm{Cs}_{2} \mathrm{AgBiBr}_{6}$ double perovskite solar cells Adv. Func. Mater. 302002342 https://doi.org/10.1002/adfm.202002342

32. K Prabhu AK Chandiran 2020 Solar energy storage in a $\mathrm{Cs}_{2} \mathrm{AgBiBr}_{6}$ halide double perovskite photoelectrochemical cell Chem. Commun. 5673297332 https://doi.org/10.1039/D0CC02743J

33. MK Kim 2015 Investigation of the magnetic properties in double perovskite $\mathrm{R}_{2} \mathrm{CoMnO}_{6}$ single crystals $(\mathrm{R}=$ rare earth: $\mathrm{La}$ to $\mathrm{Lu})$ J. Phys. Condens. Matter 27426002 https://doi.org/10.1088/0953-8984/27/42/426002

34. HS Nair T Chatterji AM Strydom 2015 Antisite disorder-induced exchange bias effect in multiferroic $\mathrm{Y}_{2} \mathrm{CoMnO}_{6}$ Appl. Phys. Lett. 106022407 https://doi.org/10.1063/1.4906204

35. RC Sahoo 2017 Antisite-disorder driven large exchange bias effect in phase separated $\mathrm{La}_{1.5} \mathrm{Ca}_{0.5} \mathrm{CoMnO}_{6}$ double perovskite J. Magn. Magn. Mater. 4288691 https://doi.org/10.1016/j.jmmm.2016.12.018

36. RI Dass JB Goodenough 2003 Multiple magnetic phases of $\mathrm{La}_{2} \mathrm{CoMnO}_{6-\delta}(0<\sim \delta<\sim 0.05)$ Phys. Rev. B. 67014401 https://doi.org/ 10.1103/PhysRevB.67.014401

37. VA Khomchenko 2006 Metamagnetic behaviour in $\mathrm{TbCo}_{0.5} \mathrm{Mn}_{0.5} \mathrm{O}_{3.06}$ perovskite J. Phys. Condens. Matter 1895419548 https:// doi.org/10.1088/0953-8984/18/42/001

38. AN Vasiliev 2008 Valence states and metamagnetic phase transition in partially B-site-disordered perovskite $\mathrm{EuMn}_{0.5} \mathrm{Co}_{0.5} \mathrm{O}_{3}$ Phys. Rev. B 77 104442 https://doi.org/10.1103/PhysRevB.77.104442

39. RP Madhogaria 2019 Evidence of long-range ferromagnetic order and spin frustration effects in the double perovskite $\mathrm{La}_{2} \mathrm{CoMnO}_{6}$ Phys. Rev. B 99104436 https://doi.org/10.1103/PhysRevB.99.104436

40. HY Choi JY Moon JH Kim YJ Choi N Lee 2017 Single crystal growth of multiferroic double perovskites: $\mathrm{Yb}_{2} \mathrm{CoMnO}_{6}$ and $\mathrm{Lu}_{2} \mathrm{CoMnO}_{6}$ Curr. Comput. Aided Drug Des. 767

41. J Blasco 2017 Magnetic order and magnetoelectric properties of $\mathrm{R}_{2} \mathrm{CoMnO}_{6}$ perovskites ( $\mathrm{R}=\mathrm{Ho}, \mathrm{Tm}, \mathrm{Yb}$, and Lu) Phys. Rev. B 96 024409 https://doi.org/10.1103/PhysRevB.96.024409

42. JT Zhang XM Lu XQ Yang JL Wang JS Zhu 2016 Origins of $\uparrow \uparrow \downarrow \downarrow$ magnetic structure and ferroelectricity in multiferroic $\mathrm{Lu}_{2} \mathrm{CoMnO}_{6}$ Phys. Rev. B 93075140 https://doi.org/10.1103/PhysRevB.93.075140

43. A Banerjee J Sannigrahi S Giri S Majumdar 2018 Magnetization reversal and inverse exchange bias phenomenon in the ferrimagnetic polycrystalline compound $\mathrm{Er}_{2} \mathrm{CoMnO}_{6}$ Phys. Rev. B 98104414 https://doi.org/10.1103/PhysRevB.98.104414

44. J Blasco 2015 Evidence of large magneto-dielectric effect coupled to a metamagnetic transition in $\mathrm{Yb}_{2} \mathrm{CoMnO}_{6}$ Appl. Phys. Lett. $107012902 \mathrm{https} / / /$ doi.org/10.1063/1.4926403

45. VS Zapf 2016 Magnetization dynamics and frustration in the multiferroic double perovskite $\mathrm{Lu}_{2} \mathrm{MnCoO}_{6}$ Phys. Rev. B 93134431 https://doi.org/10.1103/PhysRevB.93.134431

46. S Yáñez-Vilar 2011 Multiferroic behavior in the double-perovskite $\mathrm{Lu}_{2} \mathrm{MnCoO}_{6}$ Phys. Rev. B 84134427 https://doi.org/10.1103/ PhysRevB.84.134427

47. A Carr 2021 Dynamics of a fractal set of first-order magnetic phase transitions in frustrated $\mathrm{Lu}_{2} \mathrm{CoMnO}_{6}$ Phys. Rev. B $103 \mathrm{~L} 060401$ https://doi.org/10.1103/PhysRevB.103.L060401 


\section{Acknowledgements}

This work was supported by the National Research Foundation of Korea (NRF) through grants NRF-2017R1A5A1014862 (SRC program: vdWMRC center), NRF-2019R1A2C2002601, and NRF-2021R1A2C1006375.

\section{Author contributions}

N.L. and Y.J.C. designed the experiments. J.H.K. synthesized the single crystals. J.H.K., K.W.J., D.G.O., H.J.S., J.M.H., J.S.K., J.Y.M., and N.L. performed magnetization, heat capacity, dielectric constant, and polarization measurements. J.H.K., N.L., and Y.J.C. analyzed the data and prepared the manuscript. All the authors have read and approved the final version of the manuscript.

\section{Competing interests}

The authors declare no competing interests.

\section{Additional information}

Correspondence and requests for materials should be addressed to N.L. or Y.J.C.

Reprints and permissions information is available at www.nature.com/reprints.

Publisher's note Springer Nature remains neutral with regard to jurisdictional claims in published maps and institutional affiliations.

(c) (i) Open Access This article is licensed under a Creative Commons Attribution 4.0 International cc) License, which permits use, sharing, adaptation, distribution and reproduction in any medium or format, as long as you give appropriate credit to the original author(s) and the source, provide a link to the Creative Commons licence, and indicate if changes were made. The images or other third party material in this article are included in the article's Creative Commons licence, unless indicated otherwise in a credit line to the material. If material is not included in the article's Creative Commons licence and your intended use is not permitted by statutory regulation or exceeds the permitted use, you will need to obtain permission directly from the copyright holder. To view a copy of this licence, visit http://creativecommons.org/licenses/by/4.0/.

(C) The Author(s) 2021 